\title{
The quasi-optical design of the QUaD telescope
}

\author{
C. O’Sullivan ${ }^{\mathrm{a}, *}$, G. Cahill $^{\mathrm{a}}$, J.A. Murphy ${ }^{\mathrm{a}}$, W.K. Gear ${ }^{\mathrm{b}}$, J. Harris ${ }^{\mathrm{b}}$, P.A.R. Ade ${ }^{\mathrm{b}}$, \\ S.E. Church ${ }^{\mathrm{c}}$, K.L. Thompson ${ }^{\mathrm{c}}$, C. Pryke ${ }^{\mathrm{d}}$, J. Bock ${ }^{\mathrm{e}, \mathrm{f}}$, M. Bowden ${ }^{\mathrm{b}, \mathrm{c}}$, M.L. Brown ${ }^{\mathrm{g}, 1}$,

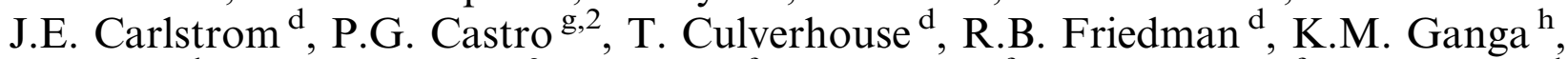 \\ V. Haynes ${ }^{\text {b }, ~ J . R . ~ H i n d e r k s ~}{ }^{\mathrm{c}, 3}$, J. Kovak ${ }^{\mathrm{f}}$, A.E. Lange ${ }^{\mathrm{f}}$, E.M. Leitch ${ }^{\text {e,f }}$, O.E. Mallie ${ }^{\mathrm{b}}$, \\ S.J. Melhuish ${ }^{\mathrm{b}, 4}$, A. Orlando ${ }^{\mathrm{b}}$, L. Piccirillo ${ }^{\mathrm{b}, 4}$, G. Pisano ${ }^{\mathrm{b}, 4}$, N. Rajguru ${ }^{\mathrm{b}, 5}$, \\ B.A. Rusholme ${ }^{\mathrm{c}, 6}$, R. Schwarz ${ }^{\mathrm{d}}$, A.N. Taylor ${ }^{\mathrm{g}}$, E.Y.S. Wu ${ }^{\mathrm{c}}$, M. Zemcov b,e,f \\ ${ }^{a}$ Department of Experimental Physics, National University of Ireland Maynooth, Maynooth, Co. Kildare, Ireland \\ ${ }^{\mathrm{b}}$ School of Physics and Astronomy, Cardiff University, Queen's Buildings, The Parade, Cardiff CF24 3AA, UK \\ ${ }^{\mathrm{c}}$ Department of Physics and Kavli Institute for Particle Astrophysics and Cosmology, Stanford University, 382 Via Pueblo Mall, Stanford, CA 94305 , USA \\ ${ }^{\mathrm{d}}$ Kavli Institute for Cosmological Physics, Department of Astronomy and Astrophysics, Enrico Fermi Institute, University of Chicago, \\ 5640 South Ellis Avenue, Chicago, IL 60637, USA \\ e Jet Propulsion Laboratory, 4800 Oak Grove Dr. Pasadena, CA 91109, USA \\ ${ }^{\mathrm{f}}$ California Institute of Technology, Pasadena, CA 91125, USA \\ ${ }^{\mathrm{g}}$ Institute for Astronomy, University of Edinburgh, Royal Observatory, Blackford Hill, Edinburgh EH9 3HJ, UK \\ ${ }^{\mathrm{h}}$ Laboratoire APCICNRS, Bâtiment Condorcet, 10, Rue Alice Domon et Léonie Duquet, 75205 Paris Cedex 13, France
}

Received 23 May 2007

Available online 20 February 2008

\begin{abstract}
We describe the optical design and performance of 'QUEST and DASI' or 'QUaD', a ground-based high-resolution experiment designed to measure the polarisation properties of the cosmic microwave background radiation. QUaD uses bolometric detectors at 100 and $150 \mathrm{GHz}$ on a $2.6 \mathrm{~m}$ Cassegrain telescope. The QUaD optics are designed to minimise systematic effects as well as to maximise sensitivity, and we report here on the comprehensive quasi-optical analysis used to achieve this design. We also present initial optical performance measurements achieved in operation, and discuss changes made to the optics to overcome some errors in the mechanical construction of the primary mirror. The QUaD experiment is now fully operational and taking world-leading data at the South Pole. (C) 2007 Elsevier B.V. All rights reserved.
\end{abstract}

PACS: 42.15.Eq; 84.40.Ba; 95.85.Bh; 98.70.Vc

Keywords: Quasioptics; Gaussian beam modes; Optical design; Feedhorn design; Cosmic microwave background

\footnotetext{
${ }^{*}$ Corresponding author. Tel.: +3531708 3953; fax: +35317083313.

E-mail address: creidhe.osullivan@nuim.ie (C. O'Sullivan).

${ }^{1}$ Current address: Cavendish Laboratory, University of Cambridge, J.J. Thomson Avenue, Cambridge CB3 OHE, UK.

${ }^{2}$ Current address: CENTRA, Departamento de Física, Edifício Ciência, Piso 4, Instituto Superior Técnico - IST, Universidade Tecnica de Lisboa, Avenue Rovisco Pais 1, 1049-001 Lisboa, Portugal.

${ }^{3}$ Current address: NASA Goddard Space Flight Centre, 8800 Greenbelt Road, Greenbelt, MD 20771, USA.

${ }^{4}$ Current address: School of Physics and Astronomy, University of Manchester, Manchester M13 9PL, UK.

${ }^{5}$ Current address: Department of Physics and Astronomy, University College London, Gower Street, London WC1E 6BT, UK.

${ }^{6}$ Current address: Infrared Processing and Analysis Center, California Institute of Technology, Pasadena, CA 91125, USA.
} 


\section{Introduction}

The cosmic microwave background (CMB) has proven to be an invaluable tool for cosmologists. Sensitive measurements of the temperature anisotropy power spectrum have made increasingly accurate estimates of the fundamental cosmological parameters. However, in addition to the temperature anisotropy signal there is an anisotropy in the linear polarisation of the CMB introduced by Thomson scattering of the photons at re-ionisation. This was detected for the first time by the degree angular scale interferometer (DASI) experiment [1]. By breaking degeneracies inherent in the temperature spectrum alone, accurate polarisation measurements by the latest generation of $\mathrm{CMB}$ experiments will lead to tighter constraints on cosmological parameters describing the density, composition, and expansion rate of the universe [2].

The Q and U extragalactic survey telescope (QUEST) at the degree angular scale interferometer (DASI) telescope [3] (the combination now called QUaD) is a ground-based experiment designed to produce the first high-resolution high signal-to-noise map of the CMB E-mode polarisation. It is also expected to make an important contribution to Bmode surveys and when combined with the four-year WMAP data will improve constraints on many cosmological parameters [4]. QUaD began observations from the South Pole in March of 2005.

Experiments such as QUaD require an in-depth understanding and control of systematic effects, particularly those that could contribute to the polarised signal. In this paper we deal specifically with the telescope and instrument optics. In Section 2 we describe the basic design criteria for the telescope and the preliminary optical configuration developed to meet them. Section 3 deals with the more detailed analysis of this design. In Section 4 we present some of the initial beam pattern measurements and finally describe some improvements made on the basis of these in Section 5 .

\section{Initial optical design}

The main criteria for the QUaD design were a high degree of axial symmetry, a large field-of-view (at least $1^{\circ}$ ) and good image quality (strehl ratio $>0.98$ ). It was decided to make simultaneous observations in two frequency bands (atmospheric transmission windows centred at approximately 100 and $150 \mathrm{GHz}$ ) to allow spectral discrimination between $\mathrm{CMB}$ and foreground signals. Pairs of orthogonal polarisation-sensitive bolometers (PSBs) [5] are used as detectors, each pair being fed by a single corrugated horn antenna. The signals from each pair are differenced to give a measure of the $Q$ and $U$ Stokes parameters. The number of feedhorns are maximised to give the high instantaneous sensitivity required for fast mapping. The resolution of the telescope $\left(\sim 4.2^{\prime}\right.$ at $150 \mathrm{GHz}$ ) was chosen to span the expected peaks in the polarisation signal.
An existing design for a $2.6 \mathrm{~m}$ parabolic primary was incorporated into an on-axis Cassegrain system with the secondary mirror supported by a Zotefoam (PPA-30) cone in order to maintain the overall symmetry of the telescope. The design allowed for a rotating achromatic half-waveplate of $100 \mathrm{~mm}$ diameter, the maximum size of sapphire crystals that could be sourced at the time. The waveplate was placed near an image of the primary mirror to minimise its diameter. The dimensions of the horn antennas were fixed by the requirement that they illuminate a truncating cold stop with an edge taper of $-20 \mathrm{~dB}$ at $12.3^{\circ}$ (a typical $F$-number for compact millimetre-wave horns). The primary mirror is under-illuminated by the feedhorns to minimise ground spillover. Spillover is further reduced by a $30 \mathrm{~cm}$ skirt around the primary mirror where the supporting foam cone for the secondary mirror is attached. The focal plane and the cold Lyot stop are located in a $4 \mathrm{~K}$ cryostat whose window dimensions were constrained by the maximum aperture of blocking filters available (200 mm).

The initial optical design work for the telescope was carried out with the software package Zemax [6] using raytracing techniques. A novel design solution (Fig. 1) using two strong field lenses was found to satisfy the above criteria and result in acceptable blockage by the $(0.225 \mathrm{~m}) \mathrm{sec}-$ ondary. The design is unusual in that the first lens is in front of the primary mirror. The lenses convert the slow beam at the focus of the telescope to the spherical F2.3 focal plane and give a field-of-view of $1.5^{\circ}$. An image of the primary mirror is formed at the cold stop where the sidelobes are truncated, resulting in well-defined beams on the primary. To minimise losses and thermal emission the lenses are anti-reflection coated and attached to the $4 \mathrm{~K}$ stage. The focal plane contains 31 pairs of PSBs; 12 operating at $100 \mathrm{GHz}$, the remaining 19 at $150 \mathrm{GHz}$.

A hole is cut in the centre of the secondary mirror. This serves to reduce the amount of power incident on the detectors which may be reflected by the secondary mirror from room-temperature parts of the cryostat. An unacceptably large hole would have to be cut if its shadow, as seen from all the feedhorns, were to exactly cover the hole in the primary. It was decided to eliminate most $(>75 \%)$ of the reflected power with a secondary hole of diameter $48 \mathrm{~mm}$ and to reduce the remainder with a baffle and flat reflecting collar around the cryostat window (Fig. 2). The reflecting collar results in a sidelobe at approximately $10^{\circ}$ off-axis.

The telescope was placed on the DASI alt-az mount so that the entire instrument can be rotated about the optical axis allowing us to characterise sources of instrumental polarisation. In the end a rotating waveplate was not installed. The whole telescope is surrounded by a shield to reflect the beam sidelobes onto the cold sky, preventing ground radiation from reaching the telescope.

Ray-tracing, although very successful at optical wavelengths, does not take into account the effects of diffraction. At millimetre wavelengths it is important to analyse the system in more detail by first determining the field at each 
a

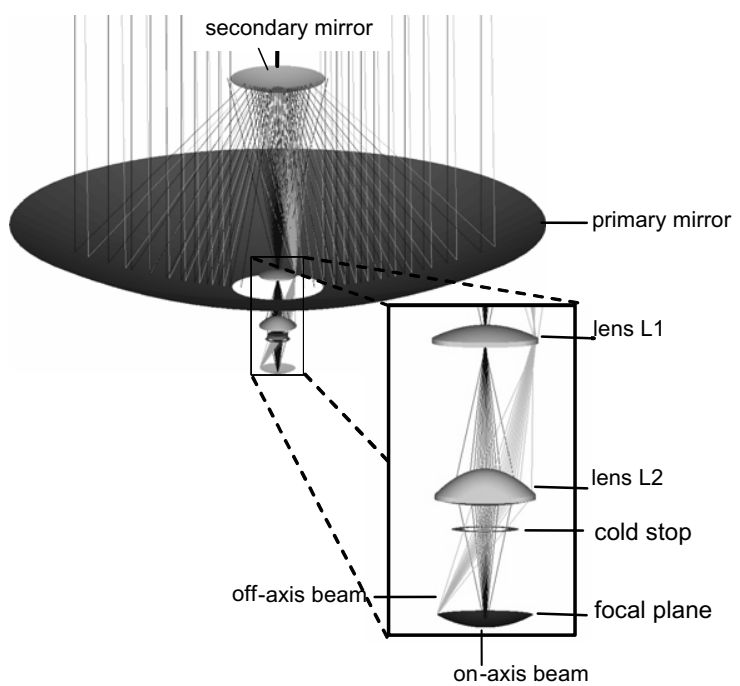

b

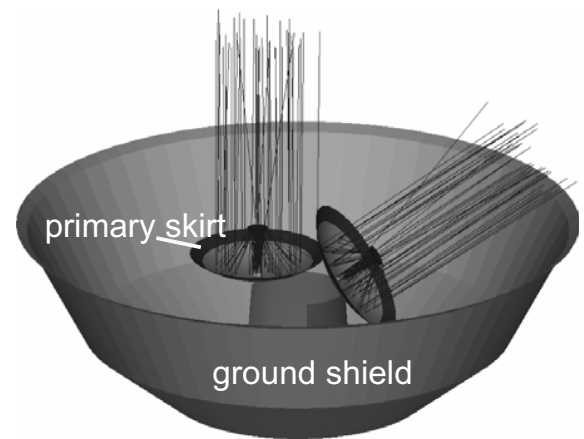

Fig. 1. (a) The QUaD telescope front-end optics. (b) The telescope (shown in two positions) with the ground shield and the skirting around the primary mirror.

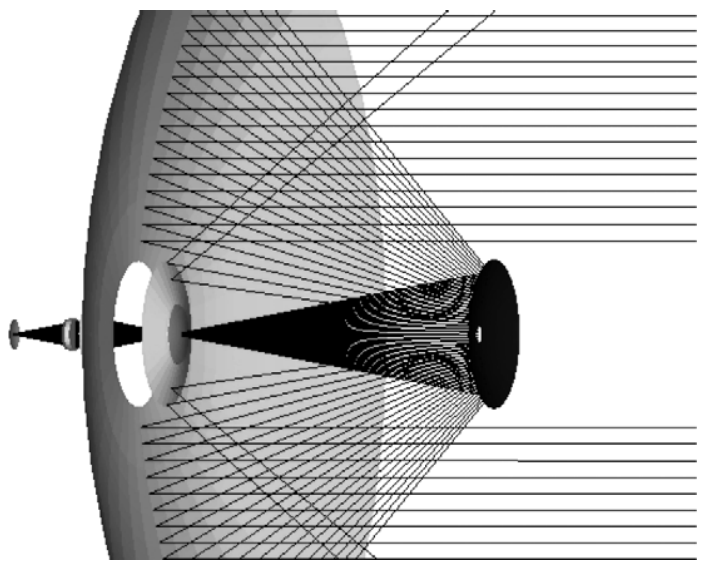

Fig. 2. Ray-tracing shows a collar design to reflect power past the secondary mirror.

of the horn apertures and then propagating these, taking account of the effect of diffraction, through a model of the telescope optics and on to the sky. We describe this more detailed analysis in the following sections.

\section{Quasi-optical analysis}

\subsection{Feedhorns}

The QUaD 100- and $150 \mathrm{GHz}$ horn design is described in detail in a paper by Murphy et al. [7] and summarised here. The QUaD horns have to produce well-controlled beams that satisfy strict criteria with regard to cross-polarisation and sidelobe level. Corrugated horns have been found to be suitable for wide-bandwidth $(\sim 33 \%$ in our case) applications and are typically designed to transmit only the linearly-polarised $\mathrm{HE}_{11}$ hybrid mode. We use cylindrically symmetric horns which transmit both orthogonal polarisations and these are coupled to the orthogonally-oriented PSBs.
The dimensions of each horn antenna are fixed by the requirement that it illuminate the cold stop with an edge taper of $-20 \mathrm{~dB}$ at $12.3^{\circ}$. Standard conical corrugated horns that meet this criterion are too long to fit into the QUaD cryostat, and so a number of alternative designs were investigated. NUI Maynooth's modelling software [8] based on a rigorous electromagnetic mode matching technique, was used to develop the prototype designs. Suitable profiled conical designs (e.g. Fig. 3) of approximately the same length were found for both frequencies and manufactured by TK Ltd. (UK). The $100 \mathrm{GHz}$ beam profiles were measured at the NUI Maynooth test range and were found to be in excellent agreement with the theoretical prediction. Above $-20 \mathrm{~dB}$, both beams are well fit by a Gaussian function with a radius of $0.6435 a$ at the horn aperture (where $a$ is the aperture radius).

For the QUaD horns the waveguide section acts as a high-pass filter defining the lower band edge of each channel; the high frequency band edges are defined by

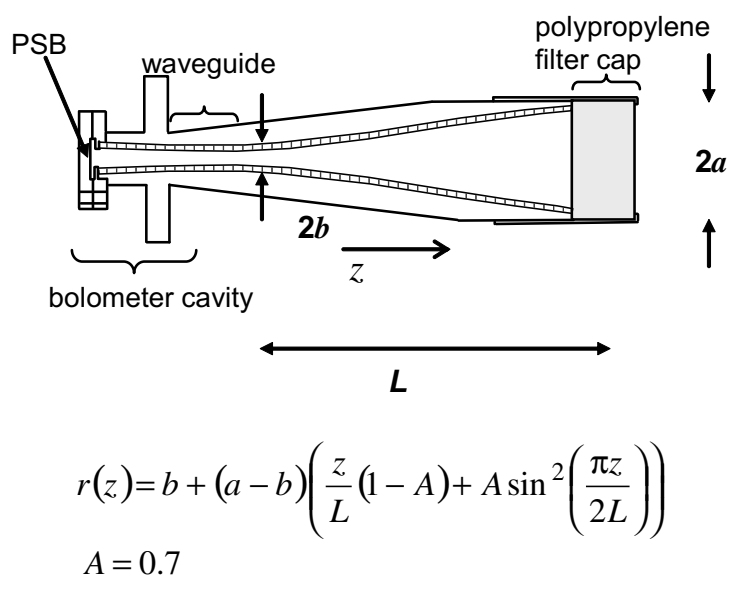

Fig. 3. Profiled conical corrugated horn design (100 GHz) for QUaD. 
hot-pressed polypropylene filters mounted at the front of each horn antenna. The mode-matching technique was used to fine-tune the horn-plus-filter design in order to get a smooth band edge at the required frequency [9].

The phase centre position of each antenna was calculated by varying its position in the optical model and maximising the on-axis gain (so the beam centre of phase curvature lies on the focal surface) [10]. The mode-matching model was used to determine the horn aperture field at five spot frequencies across each band and then these were propagated through a quasi-optical model of the telescope and added in quadrature. The resulting on-axis gains as a function of horn position are shown in Fig. 4. In order to avoid shadowing it was decided to place the front-end of each horn-plus-filter combination on the same spherical surface $23.5 \mathrm{~mm}$ in front of telescope focus. The layout of the horns on the focal surface is shown in Fig. 5. A Gaussian beam was propagated from each of these positions through the optical model of the telescope and onto the sky using the techniques described in the next section.

\subsection{Free-space propagation}

We have used paraxial modal techniques (Gaussian beam modes $[11,12]$ and the commercial software packages GLAD [13] and Zemax [6]), as well as a more rigorous vector physical optics analysis (GRASP8 [14]), to model the beam propagation through the telescope optics and include the effects of diffraction.

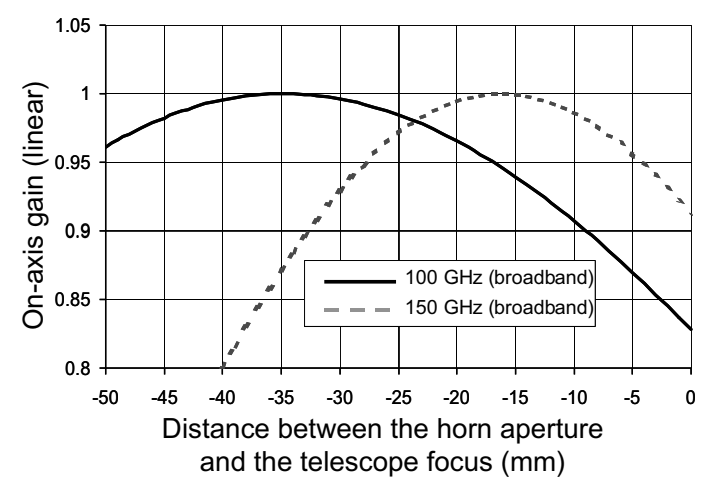

Fig. 4. Plot of on-axis gain as a function of horn position for the 100-and $150-\mathrm{GHz}$ horns. The gain is a maximum when the phase centre is located on the focal plane of the telescope.

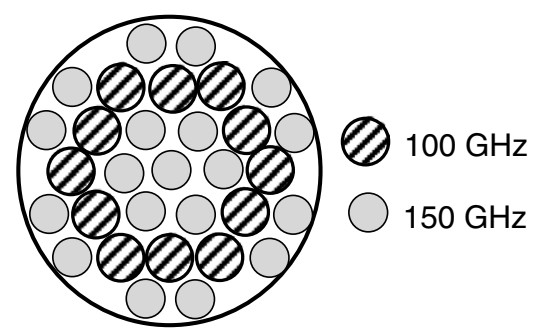

Fig. 5. Layout of the 100- and 150-GHz horns on the QUaD focal plane.
In GRASP8 a simplified model with only the primary and secondary mirrors was used. Lenses and mirrors can be analysed with the other packages and for systems such as QUaD we have found that the approximate techniques agree well with GRASP8 down to $-25 \mathrm{~dB}$ or less $[15,16]$ so long as care is taken with fast beams and near-field effects. With Zemax, for example, we could switch to raytracing when propagating from the secondary to the primary mirror where the beam is especially fast. The approximate modal techniques are computationally much less intensive and ideal for the design phase of the telescope.

\subsection{Lenses}

The QUaD design uses two re-focussing lenses between the focal surface and the telescope mirrors. Three possible materials were considered for these lenses: silicon $(n=3.4)$, quartz $(n=2.2)$ and HDPE $(n=1.583$, a value we obtained from room-temperature measurements of our sample and an extrapolation to $4 \mathrm{~K}$ based on published warm and cold values). An important consideration for an experiment such as QUaD is the introduction of any polarisation effects. On propagating out from the focal plane, each beam will travel through four vacuum/material interfaces (the two lenses). At each interface there is a difference in transmission for radiation polarised perpendicular and polarised parallel to the plane of incidence [17]. This difference increases with increasing angle of incidence and has the effect of imposing a measured polarisation on an unpolarised source (even for anti-reflection coated lenses because of the wide-bandwidth of the signal). It is extremely important that this effect is minimised and carefully characterised. Table 1 lists the percentage power transmission (see also Fig. 6) and instrumental polarisation for the most off-axis 100- and 150-GHz QUaD pixel. For single-layer anti-reflection coating HDPE was found to be the most suitable lens material.

The anti-reflection coating on the lenses was chosen as a $\lambda / 4$-thick layer of material of refractive index as close as possible to $\sqrt{ } n$ with $\lambda$ taken as the wavelength midway between the two observing bands. Since $3 \times \lambda / 4$ for the centre of the $100 \mathrm{GHz}$ band is close to $5 \times \lambda / 4$ for the centre of the $150 \mathrm{GHz}$ band, this was another option considered for the coating thickness. However, in this case, the average transmission over each band was found to be poorer (Fig. 7).

Table 1

Percentage power transmission $(T)$ and instrumental polarisation (Pol) through the lenses for the most-off-axis QUaD pixels

\begin{tabular}{|c|c|c|c|c|c|c|}
\hline \multirow[t]{2}{*}{ Frequency $(\mathrm{GHz})$} & \multicolumn{2}{|c|}{ HDPE } & \multicolumn{2}{|c|}{ Quartz } & \multicolumn{2}{|l|}{ Silicon } \\
\hline & $T(\%)$ & Pol (\%) & $T(\%)$ & Pol (\%) & $T(\%)$ & Pol $(\%)$ \\
\hline 100 & 97.6 & 0.3 & 92.1 & 0.3 & 82.1 & 3.6 \\
\hline 150 & 95.9 & 1.3 & 84.6 & 2.9 & 63.5 & 2.2 \\
\hline
\end{tabular}

Calculations were made for three different lens materials using Zemax. 


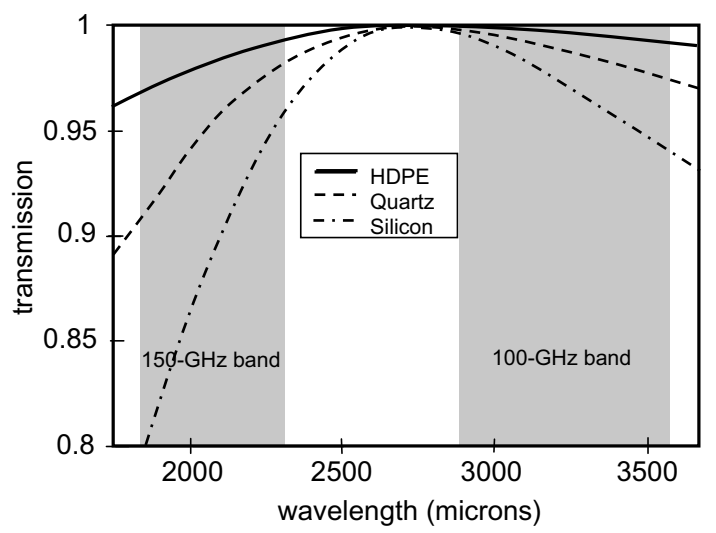

Fig. 6. Transmission as a function of wavelength for three possible lens materials (the AR coating thickness was chosen for a wavelength between the two observing bands).

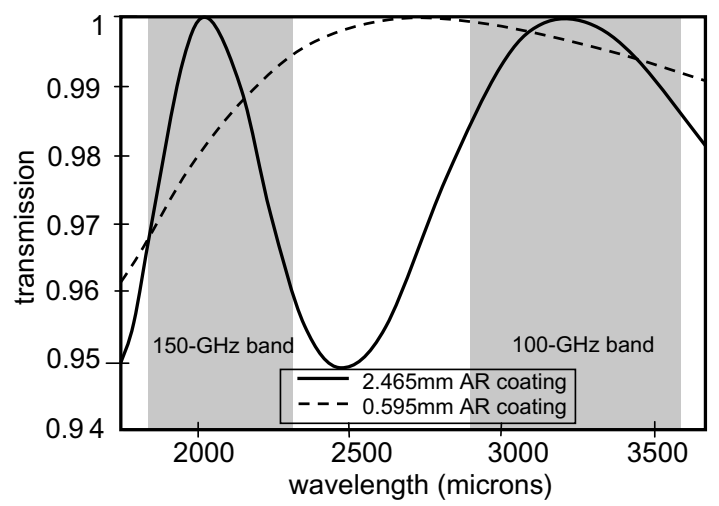

Fig. 7. Transmission as a function of wavelength for an AR coating matched to the centres of both bands $(2465 \mathrm{~mm})$ and matched to a wavelength between the two bands $(0.595 \mathrm{~mm})$.

\subsection{Mirrors}

The primary and secondary mirrors were designed to be parabolic and hyperbolic, respectively. However, measurements at the observing site found a systematic deviation from the primary mirror design surface (Fig. 8). This deviation is well-modelled by a Zernike astigmatism term

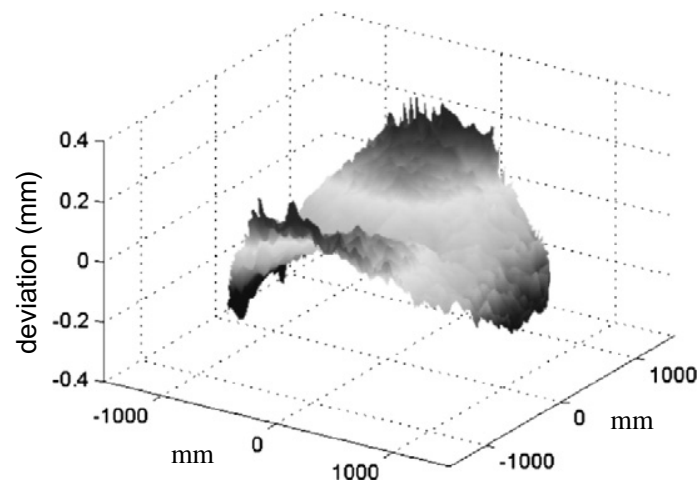

Fig. 8. Measured deviations (in $\mathrm{mm}$ ) from the design parabolic surface of the primary mirror. superimposed on the original parabola. (GRASP8 can take the measured data points as input.)

The warp on the primary mirror results in a different best-focus position in orthogonal directions. Fig. 9 shows the predicted beamwidth as a function of mirror separation, our most critical parameter. It is possible to adjust this separation so that the beam is symmetric (although not for all pixels simultaneously) but the beams will be wider than for a perfect mirror. For all other separations the beams are predicted to be elliptical in shape and any variation in the mirror separation will result in beams of varying ellipticities.

Near-field diffraction effects were found to produce some power in the geometrical shadow of the secondary mirror hole (Fig. 10). This 'Poisson spot' effect is not predicted by ray-tracing but for QUaD the amount of power in the on-axis spike is very small.

\subsection{Far-field beam pattern predictions}

An example of the beam patterns for the on-axis pixel and one of the off-axis pixels is shown in Fig. 11 for a perfectly parabolic primary mirror. The predictions made

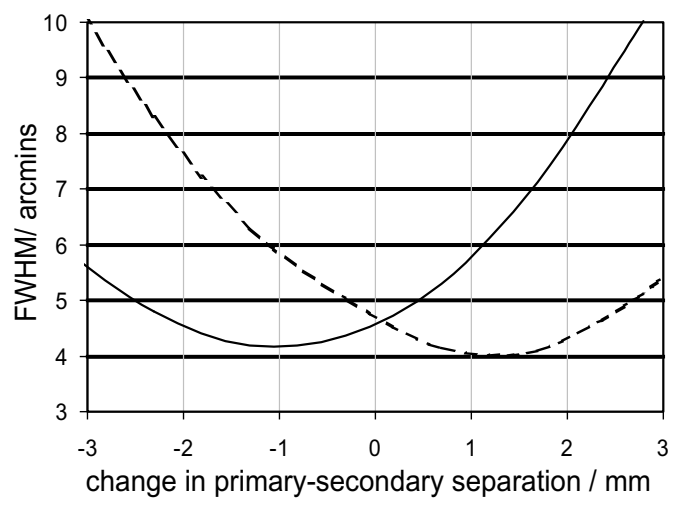

Fig. 9. Beamwidth on the sky in orthogonal directions for different mirror separations.

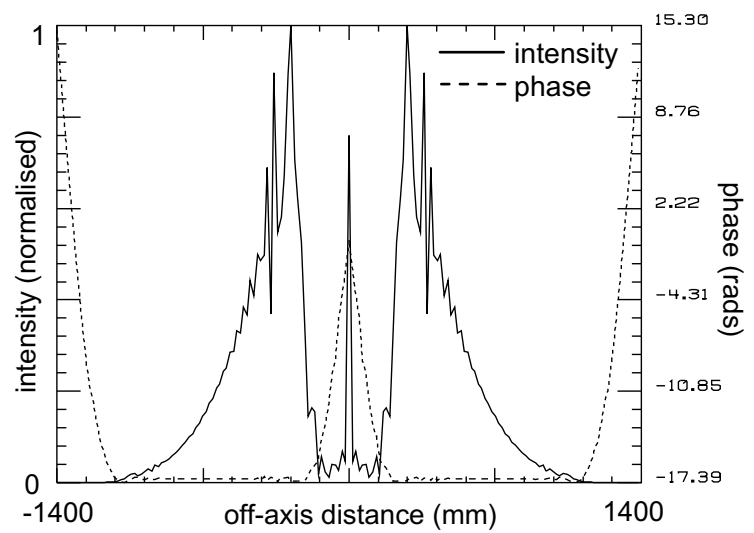

Fig. 10. GLAD prediction of the beam pattern at the primary $(r=24 \mathrm{~mm}$ hole, $\lambda=2.0 \mathrm{~mm}$ ). 

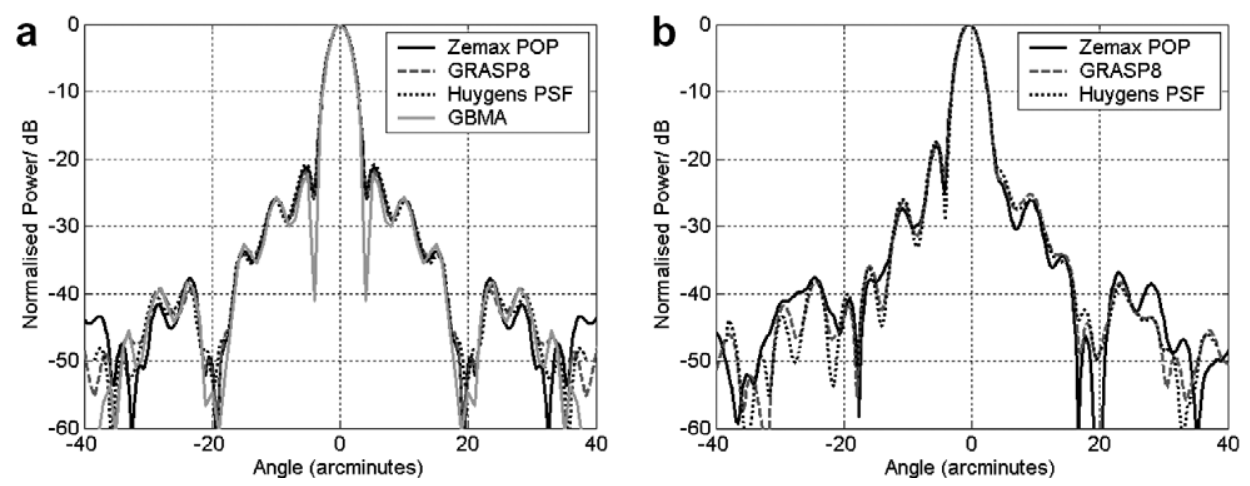

Fig. 11. Cuts through far-field beam patterns of: (a) the on-axis pixel and (b) an off-axis pixel calculated using different techniques and software packages. (GMBA $=$ Gaussian beam mode analysis, Huygen's PSF is a technique used by Zemax that does not consider diffraction until the last optical element.)

using the various techniques and software packages agree well to $-40 \mathrm{~dB}$. Using the exact horn beam patterns calculated from the mode-matching analysis rather than the Gaussian approximation only affected the beam patterns below the $-25 \mathrm{~dB}$ level.

The cross-polar power in the far-field beam pattern (Fig. 12) was investigated using Zemax and GRASP8. Both packages predict that the telescope mirrors introduce only very low levels of cross-polarisation. For the most off-axis pixel, the peak cross-polar power level was $42.5 \mathrm{~dB}$ below that of the co-polar power.

The beam patterns for the warped primary mirror were also calculated for different mirror separations. The separation would be expected to vary with any thermal expansion or contraction of the foam cone, for example. Fig. 13a-e show that a variation of a few millimetres would have a significant effect on the beam patterns.

\section{Beam pattern measurements}

The QUaD beam patterns were measured by scanning the bright compact $\left(<1^{\prime}[18]\right)$ galactic HII region RCW38. Examples of the resulting raster maps are shown in Fig. 14a. As predicted, the beams were elliptical in shape with the widths along the major and minor axes differing by up to a factor of 2 .

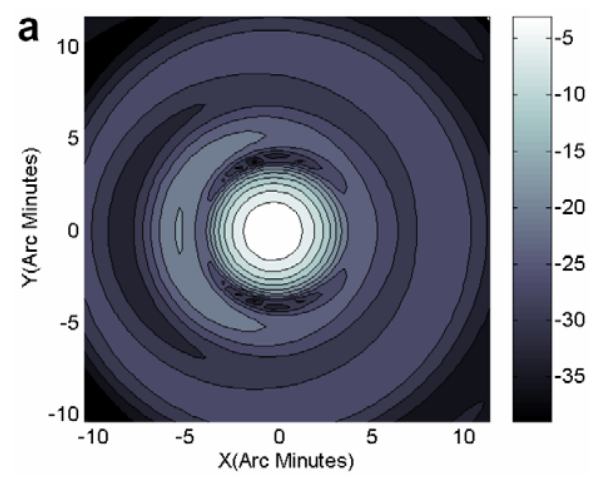

The optimisation function in Zemax was used to vary the model mirror separation and the orientation of the primary mirror until the beamwidths and orientations predicted matched those measured. A separation $2.5 \mathrm{~mm}$ smaller than the nominal position was found to fit the data well (Fig. 15a). It was decided to re-focus the telescope (move the secondary further from the primary by $1.7 \mathrm{~mm}$ ) to make as many of the beams as possible symmetric. The post re-focus beams are shown in Fig. 14b.

The re-focus maximised the number of symmetrical beams but the inner ring of $150 \mathrm{GHz}$ pixels remained elliptical (major-to-minor ratio of up to 1.2) and the ellipticity of the pixels still varied with any change in mirror separation.

Although we found our optical model to be very successful in modelling the beam shapes, a more sophisticated model is required to reproduce all the features observed during our calibration observations. For each pixel we found that the beam centres of the orthogonal polarisations were displaced, by up to $0.2^{\prime}$ in the case of the most off-axis $150 \mathrm{GHz}$ pixels. We would need to, for example, introduce birefringence into our window or lenses in order to predict such an effect. Measurements of birefringence in plastics have been made elsewhere, e.g. [19], but values are very dependent on sample production methods. We also found a sharp radial sidelobe (below about $-60 \mathrm{~dB}$ ),

Fig. 12. Normalised (a) co- and (b) cross-polar far-field beam patterns (scale in $\mathrm{dB}$ ) for the most off-axis pixels calculated using GRASP8 (no lenses included in the model). The peak cross-polar power level was $\sim 42.5 \mathrm{~dB}$ below the on-axis co-polar value. 

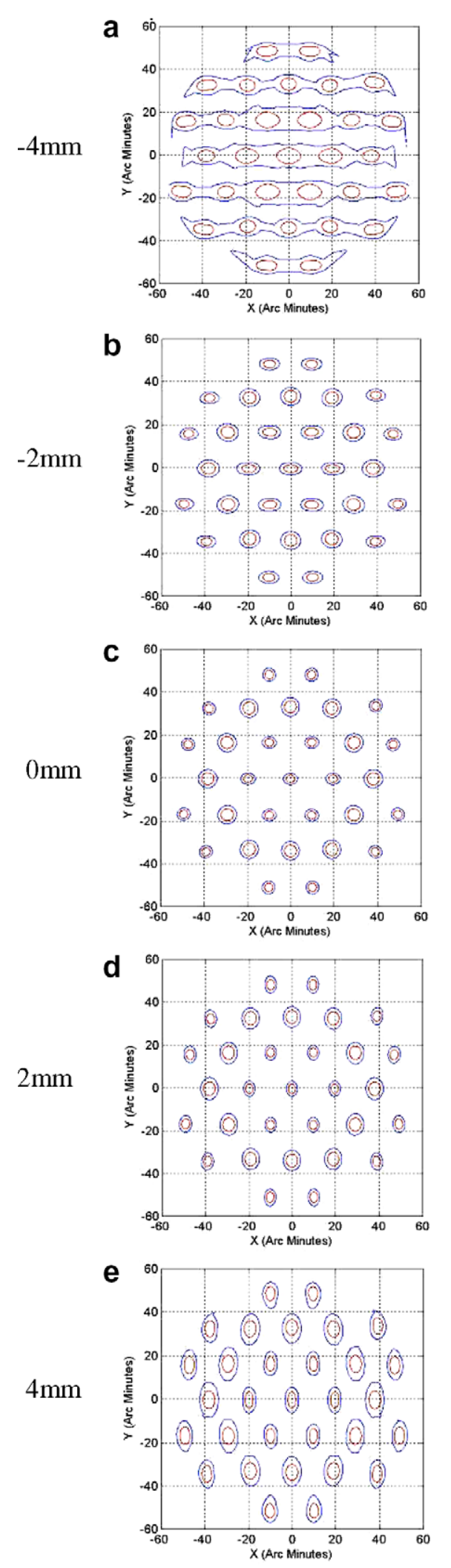

with hyperbolic secondary
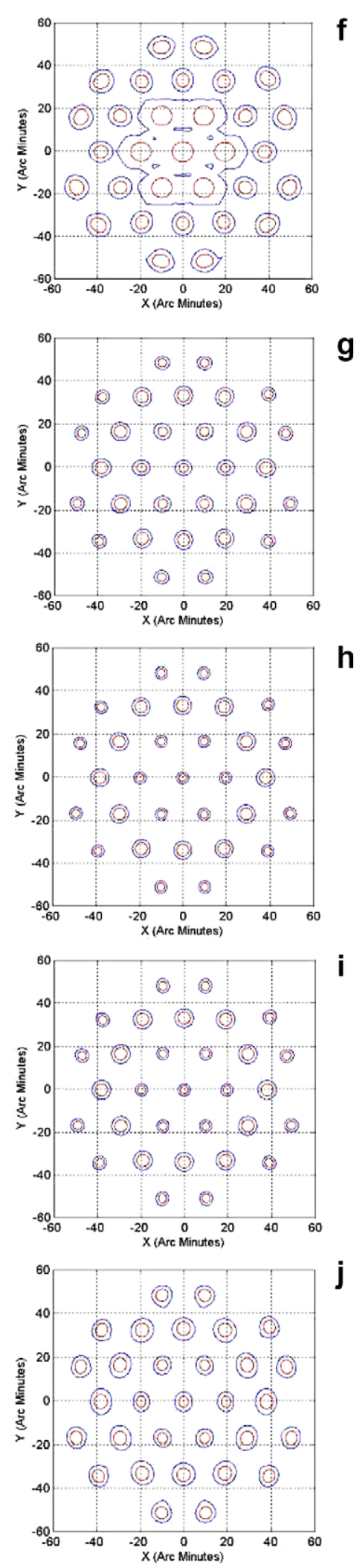

with modified secondary

Fig. 13. Predicted QUaD beam patterns as a function of the mirror separation. Plots (a)-(e) are for the distorted primary and a perfect hyperbolic secondary, (f)-(j) show the situation when the secondary has a compensating second-order Zernike term added. (The $-3 \mathrm{~dB}$ and $-9 \mathrm{~dB}$ contours are shown.) 
a
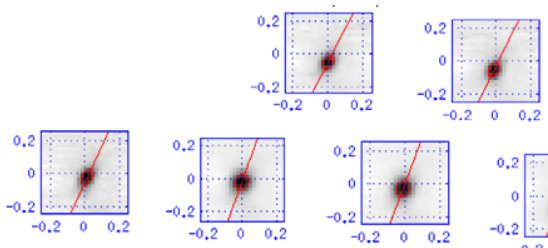

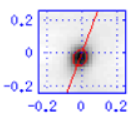

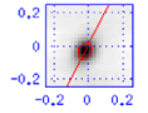

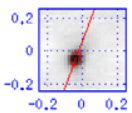

\begin{tabular}{c|c|c|c|c|c|c|c|}
0.2 & \\
-0.2 & & \\
-0.2 & 0 & 0.2
\end{tabular}

\begin{tabular}{c|ccc|}
0.2 & & & \\
\hline-0.2 & & & \\
\hline-0.2 & 0 & 0.2 \\
\hline
\end{tabular}

\begin{tabular}{cccc|}
\hline 0.2 & & & \\
\hline & $\ldots$ & & \\
\hline-0.2 & $\ldots$ & & \\
\hline-0.2 & 0 & 0.2 \\
\hline
\end{tabular}

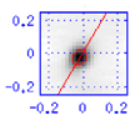
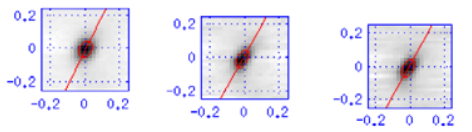

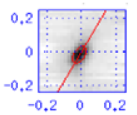

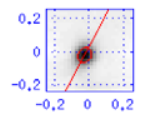

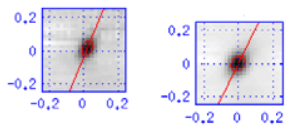
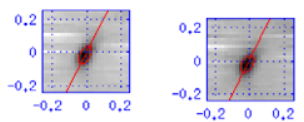

\begin{tabular}{llllll}
\hline & 0.2 & & & & \\
\hline & & & & \\
-0.2 & & & & \\
\hline-0.2 & 0 & 0.2 \\
\hline
\end{tabular}
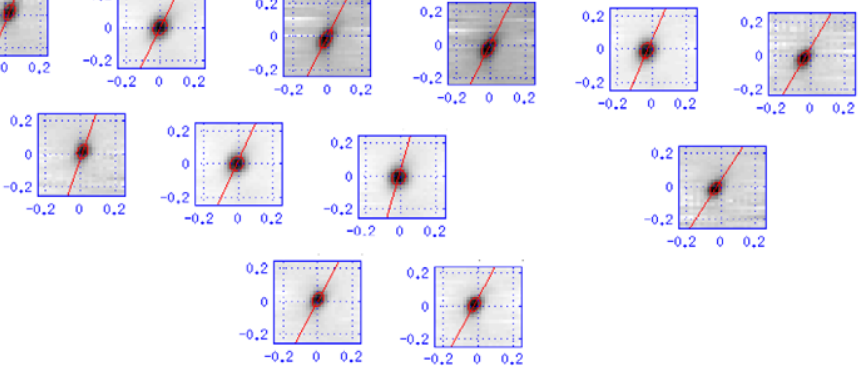

b
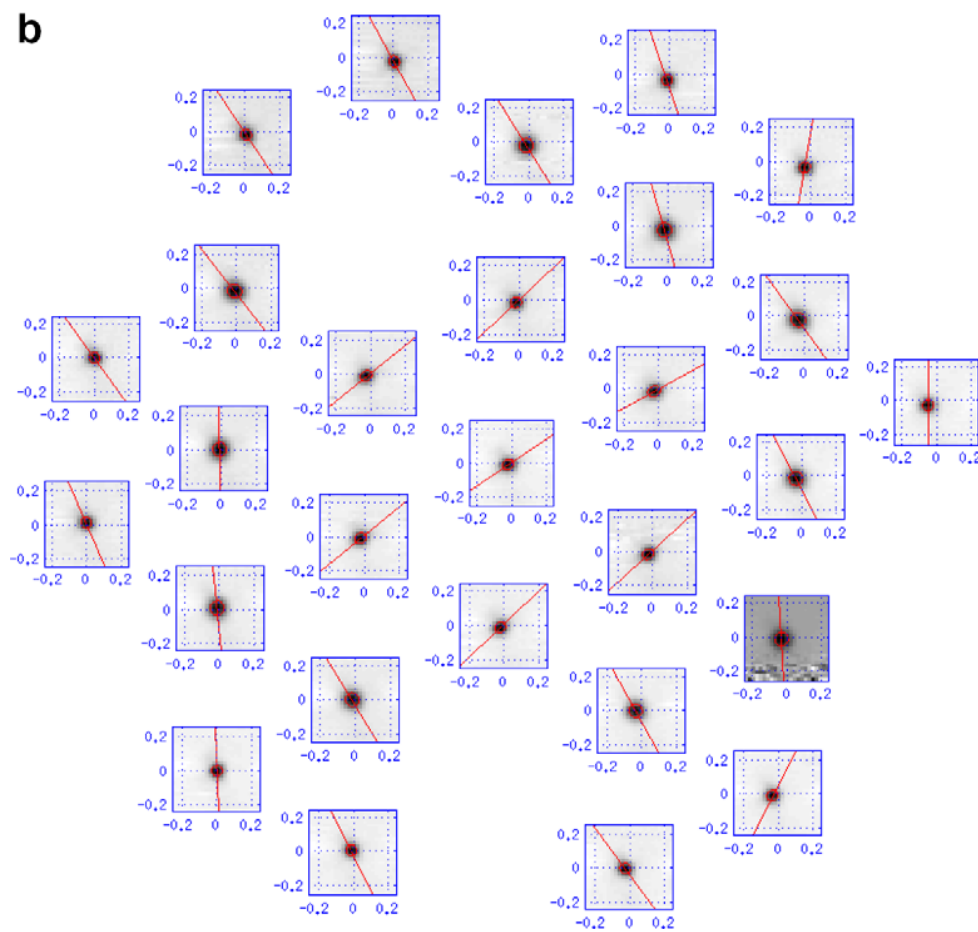

Fig. 14. Season 1 beam maps made by scanning the compact galactic source RCW38 [18]: (a) before and (b) after re-focussing of the telescope. For each pixel the best-fit elliptical Gaussian and its orientation are shown (units are degrees).

appearing mainly in the outer $150 \mathrm{GHz}$ beams, associated with stray radiation entering the system directly from the front edge of the cryostat, possibly due to diffraction from filter rims. Adding a cylindrical eccosorb forebaffle removed this sidelobe at angles greater than about $14^{\circ}$ from the main lobe but at the cost of increasing the thermal loading of the outer pixels. It was decided not to use such a baffle.

\section{Season two modifications}

Improving the symmetry of the beams was considered a high priority for the second QUaD observing season. Since the error on the primary can be well-modelled by a loworder Zernike polynomial, it was decided to compensate for the distortion by manufacturing a new secondary mirror and including such a term. The beam predictions with 

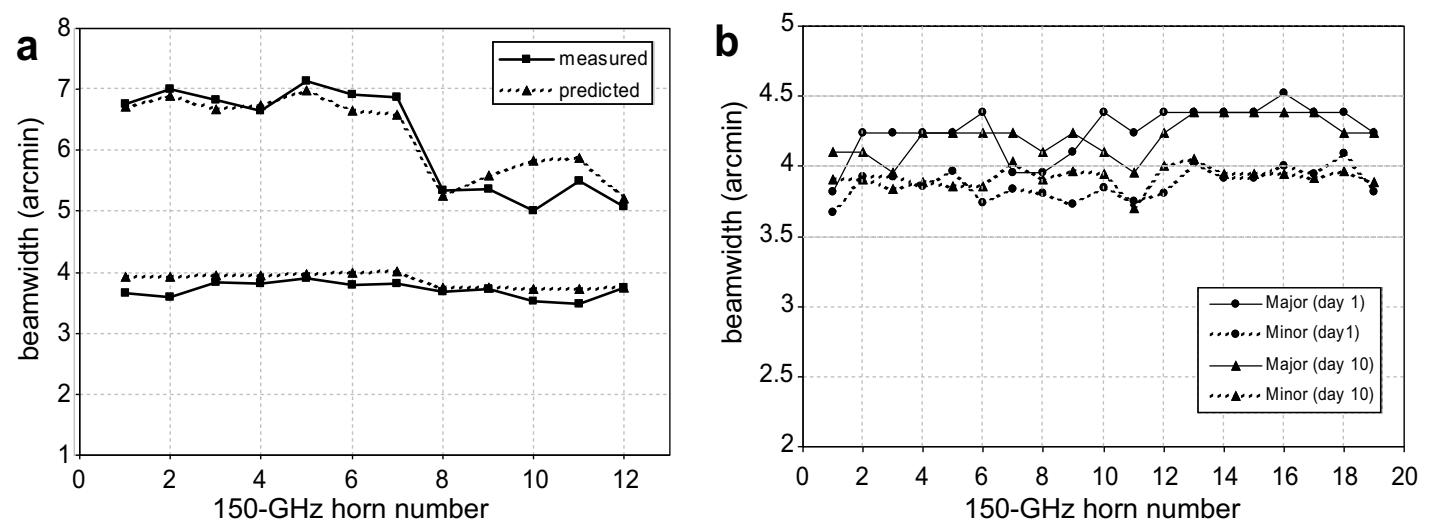

Fig. 15. 150-GHz beam FWHM along the major and minor axes of the best-fit ellipse in (a) season 1 and (b) season 2 after the new secondary mirror was installed. (The measured beam widths of the first 12 of the $150-\mathrm{GHz}$ pixels were fit to the Zemax model in season 1 . The two sets of measurements in (b) were taken 10 days apart and for two different orientations of the telescope.)
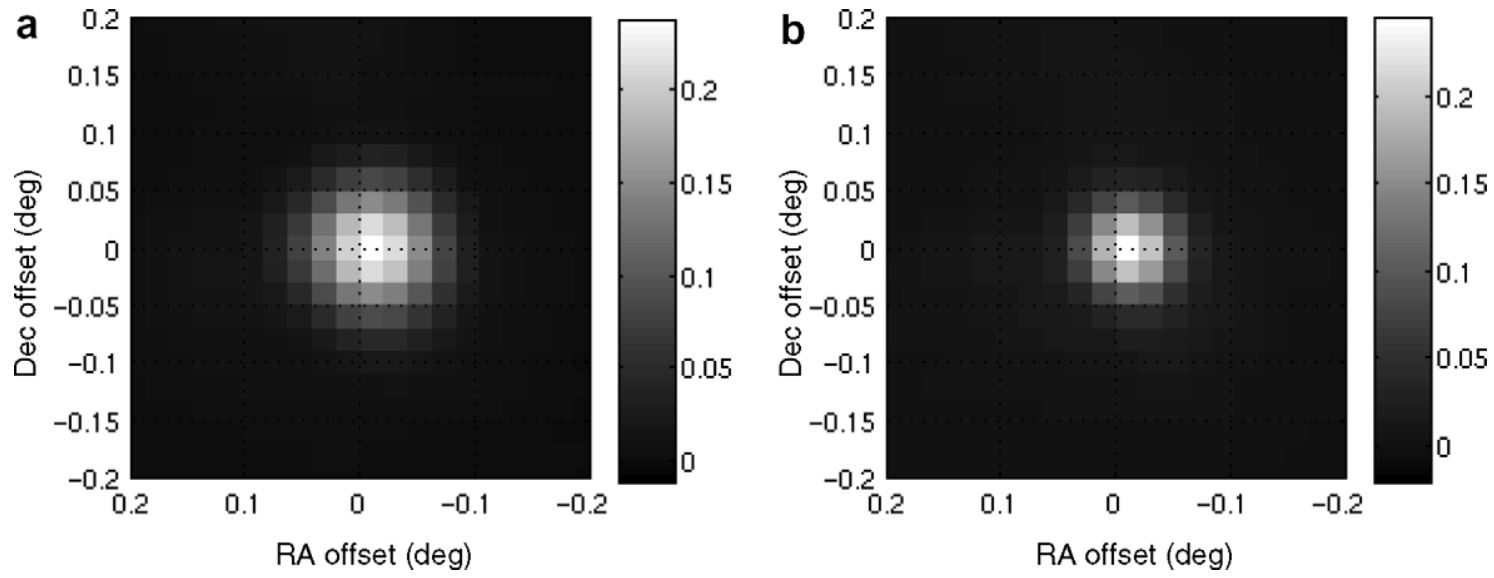

Fig. 16. (a) $100 \mathrm{GHz}$ and (b) $150 \mathrm{GHz}$ beam maps made using RCW38 in March 2006. Measurements from pixels at the same frequency have been added.

this new secondary mirror are presented in Fig. 13f-j. The beams are predicted to be significantly more symmetrical over a large range of mirror separations, and this has indeed been found in practice.

Since variations in external temperature have been found to have a significant effect upon the mirror separation, it was also decided to fit a mechanism to the telescope to allow dynamic repositioning of the secondary along the optical axis. The secondary is first mounted at the optimum distance from the primary and a further $\pm 10 \mathrm{~mm}$ of travel is allowed by the linear actuators of the new focus mechanism.

The measured FWHM along the major and minor axis of the season two beams are plotted in Fig. 15b. The modifications to the optics have greatly improved both the symmetry and reproducibility of the beams. An example of the season two beam maps, made using scans of RCW38, is presented in Fig. 16, and in Fig. 17 we show a total- and polarised-intensity map of the moon made in August 2005, a few days from Full Moon. The expected radial polarisation pattern (e.g [20]), with polarisation increasing

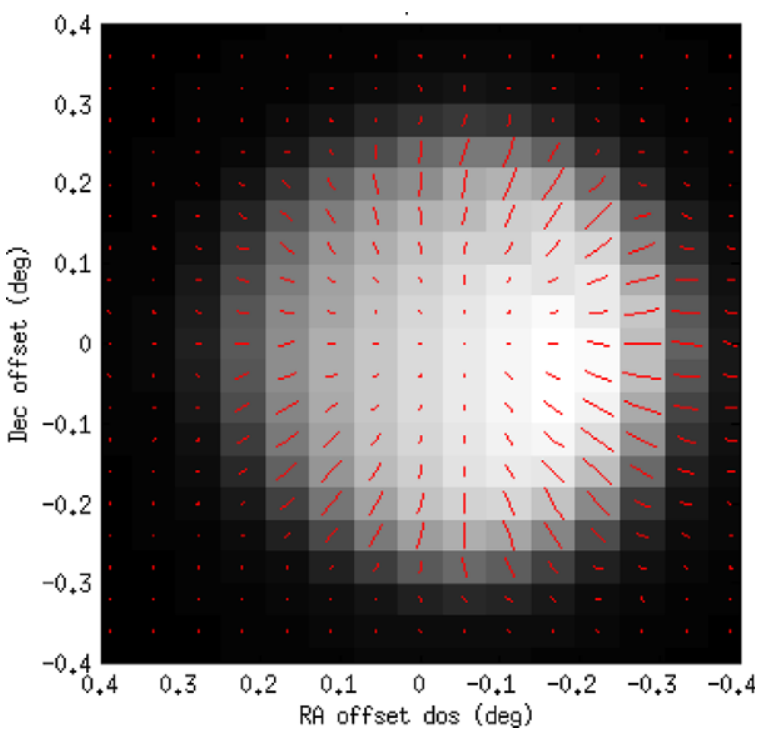

Fig. 17. Total intensity and polarisation vector map of the Moon made by QUaD on August 15th and 16th 2005. The primary mirror was partially in the shadow of the ground shield during this observation. 
towards the rim, is clearly seen. A comprehensive description of the entire instrument design and calibration can be found in [21] and the first-season's CMB results in [22].

\section{Summary and conclusions}

In this paper we have described the design and analysis of the front-end optics for QUaD, a ground-based CMB polarisation experiment. Ray-tracing techniques were used to develop the initial Cassegrain configuration based on an existing design for a $2.6 \mathrm{~m}$ parabolic primary. A novel solution was found which satisfied a strict set of criteria with regard to sensitivity, field of view and cross-polarisation.

This design was then further analysed using techniques more suitable for millimetre wavelengths. We compared the predictions of software that use paraxial modal techniques to model diffraction (GBM, GLAD, ZEMAX) with those from a more rigorous, and computationally intensive, vector physical optics approach (GRASP8). We found that once care was taken with fast beams the results compared well for our system. Because of their speed and flexibility, the more approximate modal techniques were found to be particularly useful in the design phases of the project.

We described results from the diffraction analysis of the telescope, tracing beams from the focal plane feedhorns, through the lenses, mirrors and finally out onto the sky. The surface of the primary mirror was measured and found to have a small systematic distortion which our model predicts will result in elliptical beams, the ellipticities of which depend sensitively on the separation of the primary and secondary mirrors. Beam maps made from raster scans of the compact galactic source RCW38 showed that this was indeed the case. The optical model was used to re-focus the telescope taking the primary distortion into account. The secondary mirror was then re-designed for the second season of observing to compensate for the error on the primary. The season-two beam patterns were found to be significantly more symmetric and stable.

\section{Acknowledgements}

We would like to thank NUI Maynooth and Science Foundation Ireland for supporting this work financially. The QUaD instrument is funded by the National Science Foundation (USA) and the Particle Physics and Astronomy Research Council (UK).

\section{References}

[1] J. Kovac, E.M. Leitch, C. Pryke, J.E. Carlstrom, N.W. Halverson, W.L. Holzapfel, Detection of polarization in the cosmic microwave background using DASI, Nature 420 (2002) 772.
[2] M. Zaldarriaga, D.N. Spergel, U. Seljak, Microwave background constraints on cosmological parameters, ApJ. 488 (1997) 1.

[3] S. Church et al., QUEST on DASI: a South-Pole CMB polarization experiment, New Astron. Rev. 47 (2003) 1083-1089.

[4] M. Bowden et al., Scientific optimisation of a ground-based CMB polarization experiment, MNRAS 349 (1) (2004) 321-335.

[5] W.C. Jones, R. Bhatia, J.J. Bock, A.E. Lange, A polarization sensitive bolometric receiver for observations of the cosmic microwave background, in: T.G. Phillips, J. Zmuidzinas (Eds.), Proceedings of the SPIE, vol. 4855, p. 227, 2003.

[6] ZEMAX Development Corporation, Zemax-EE, http://www.zemax. $\mathrm{com} /$.

[7] J.A. Murphy, E. Gleeson, G. Cahill, W. Lanigan, C. O'Sullivan, E. Cartwright, S.E. Church, J. Hinderks, E. Kirby, K. Thompson, B.W.K. Rusholme, B. Maffei, P.A.R. Ade, C. Tucker, B. Jones, Millimeter-wave profiled corrugated horns for the QUaD cosmic background polarization experiment, Int. J. IR Millimeter Waves 26 (4) (2005) 505-523.

[8] J.A. Murphy, R. Colgan, C. O'Sullivan, B. Maffei, P. Ade, Radiation patterns of multi-moded corrugated horns for far-IR space applications, Infrared Phys. Technol. 42 (2001) 515-528.

[9] E. Gleeson, J.A. Murphy, B. Maffei, W. Lanigan, J. Brossard, G. Cahill, E. Cartwright, S.E. Church, J. Hinderks, E. Kirby, C. O'Sullivan, Corrugated waveguide band edge filters for CMB experiments in the far infrared, Infrared Phys. Technol. 46 (6) (2005) 493-505.

[10] E. Gleeson, J.A. Murphy, B. Maffei, Phase centres of far infrared multi-moded horn antennas, Int. J. Infrared Millimetre Waves 23 (5) (2002) 711-730.

[11] M.L. Gradziel, D. White, N. Trappe, R.J. Mahon, T.J. Finn, S. Withington, J.A. Murphy, C.M. O'Sullivan, Modelling of millimetrewave and Terahertz imaging systems, Proceedings of the SPIE 5619 (2004) 154-165.

[12] P. Goldsmith, Quasioptical systems: Gaussian beam quasioptical propagation and applications, IEEE Press, New York, 1997.

[13] Applied Optics Research, GLAD, http://www.aor.com.

[14] TICRA Engineering Consultants, GRASP8, http://www.ticra.dk.

[15] C. O'Sullivan, E. Atad-Ettedgui, W. Duncan, D. Henry, W. Jellema, J.A. Murphy, N. Trappe, H. van de Stadt, S. Withington, G. Yassin, Far-IR optics design and verification, Int. J. IR Millimeter Waves 23 (2002) 7.

[16] G. Yassin, S. Withington, C. O'Sullivan, J.A. Murphy, T. Peacocke, W. Jellema, P. Wesselius, Electromagnetic simulations of submillimetre-wave systems, in: Proceedings of 13th International Symposium on Space Terahertz Technology, Harvard, USA, 2002.

[17] M. Born, E. Wolf, Principles of Optics, Cambridge University Press, 1999.

[18] K. Coble et al., Observations of Galactic and Extra-galactic Sources From the BOOMERANG and SEST Telescopes, arXiv:astro-ph/ 0301559v1, January 2003.

[19] A. Boldizar, S. Jacobsson, S. Hård, Far infrared birefringence versus other orientational measurements of high-pressure injection-molded high-density polyethylene, J. Appl. Polym. Sci. 36 (7) (1988) 15671581.

[20] E.M. Leitch, J.M. Kovac, C. Pryke, J.E. Carlstrom, N.W. Halverson, W.L. Holzapfel, M. Dragovan, B. Reddall, E.S. Sandberg, Measurement of polarization with the degree angular scale interferometer, Nature 420 (2002) 763-771

[21] J. Hinderks et al., ApJ Supplement, submitted for publication.

[22] P.A.R. Ade et al., First season QUaD CMB temperature and polarization power spectra, ApJ, accepted for publication (2008). 